\title{
Duality-based approximation for the critical point of the square lattice Ising ferromagnet within Tsallis statistics
}

\author{
L.R. da Silva ${ }^{\mathrm{a}, \mathrm{b}, *}$, H.E. Stanley \\ ${ }^{2}$ Center for Polymer Studies and Department of Physics, Boston University, Boston, MA 02215. USA \\ ${ }^{b}$ Departamento de Física, Universidade Federal do Rio Grande do Norte, Campus Universitário, \\ C.P. 1641,59072-970-Natal-RN, Brazil
}

Received 25 January 1996

\begin{abstract}
Within the generalized thermostatistics of Tsallis, we propose for the spin- $\frac{1}{2}$ Ising ferromagnet a transmissivity variable which extends that defined by Tsallis and Levy for thermal magnetic systems. By using this generalized transmissivity as well as duality arguments, we calculate the $q$-dependence of the critical temperature corresponding to the square lattice, where $q$ is the entropic index ( $q=1$ reproduces standard thermostatistics). Our approximate results are compared with those previously obtained using renormalization group and mean-field approximation.
\end{abstract}

PACS: $02.50 . \mathrm{K} ; 05.70 ; 65.50 ; 75.10$

\section{Introduction}

The thermal transmissivity [1] (see also [2] and references therein) is a convenient variable introduced to treat classical discrete spin magnetic systems. One of the advantages of this variable arises from the fact that, in various standard situations, it maps the $[0, \infty]$ temperature interval into the $[0,1]$ interval. Its key strength is that it provides a geometrical interpretation [3] for the flow of thermal information (we shall illustrate this below for the spin- $-\frac{1}{2}$ Ising ferromagnet). Finally, it enables a simple approach for arbitrary finite clusters, the bonds of which are associated with arbitrary coupling constants (this is particularly convenient within real-space renormalization group).

For the $\lambda$-state Potts Hamiltonian defined by

$$
\mathscr{H}=-\lambda J \sum_{\langle i, j\rangle} \delta_{\sigma_{i}, \sigma_{i}}\left(\sigma_{i}=1,2, \ldots, \lambda\right)
$$

*Corresponding author. 
$\left(\langle i, j\rangle\right.$ denotes the first neighbors; $\delta_{\sigma_{i}, \sigma_{j}}$ refers to Kroenecker delta function) the transmissivity is defined by (see $[1,2]$ and references therein)

$$
t \equiv \frac{1-e^{-\lambda J / k_{B} T}}{1+(\lambda-1) e^{-\lambda J / k_{B} T}},
$$

where $k_{B}$ is the Boltzmann constant and $T$ is the temperature.

In the case we shall focus on here (Ising model), $\lambda=2$. Hence

$$
t=\frac{1-e^{-2 J / k_{B} T}}{1+e^{-2 J / k_{B} T}}=\tanh \left(\frac{J}{k_{B} T}\right) .
$$

Recently, Tsallis [4] proposed a generalized entropy which leads to nonextensive statistical mechanics and thermodynamics. This generalized entropy is defined as follows:

$$
S_{q} \equiv k_{B} \frac{1-\sum_{i=1}^{W} p_{i}^{q}}{q-1} \quad(q \in \mathscr{R}),
$$

where $\left\{p_{i}\right\}$ are the occurrence probabilities of the $W$ microstates of the system. The $q \rightarrow 1$ limit recovers the usual Shannon expression, i.e.,

$$
S_{1}=-k_{B} \sum_{i=1}^{W} p_{i} \ln p_{i}
$$

Several results of the literature have been extended using the Tsallis generalized statistics. Among them, we have Lévy-like [5] and correlated [6] anomalous diffusion, self-gravitating systems $[7,8], d=2$ turbulence [8], ferrofluid-like systems [9], hydrogen atom [10] and background cosmic radiation [11]. Also, it has been successfully used in the context of simulated-annealing optimization techniques [12], and a number of related aspects have been studied [13-26].

The influence of the nonextensitivity on phase transitions is certainly a field which should be explored. In particular, questions like the $q$ dependence of critical points and critical exponents are wide open. The present work provides some clues along this line.

In the present work we focus on a simple magnetic system. Following the previous works $[1,2]$, we extend the transmissivity (denoted $t(q)$ ), from $q=1$ to all $q$, within the framework of Tsallis statistics, thus generalizing the concept originally introduced within Boltzmann-Gibbs statistics.

In Section 2 we recall the relevant transmissivity concepts within the BoltzmannGibbs statistics $(q=1)$; in Section 3 we present the Tsallis statistics (for all $q$ ). Finally, we conclude in Section 4.

\section{Boltzmann-Gibbs statistics $(q=1)$}

Let us briefly review the known transmissivity procedure for calculating the critical temperature within Boltzmann-Gibbs statistics (see Ref. [4]). 


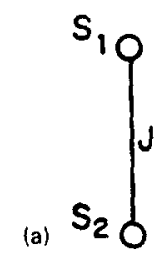

(b)

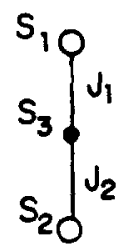

(c)

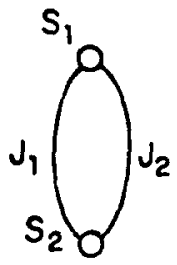

Fig. 1. Ising clusters for the (a) single bond, (b) two-series array, and (c) two-parallel array.

Consider the graphs in Fig. 1. The Boltzmann factor associated with Fig. 1(a) is given by

$$
e^{-\beta \mathscr{C}_{12}}=\mathrm{e}^{\beta J S_{1} S_{2}}=\cosh (\beta J)\left[1+\tanh (\beta J) S_{1} S_{2}\right] \equiv A\left(1+\frac{B}{A} S_{1} S_{2}\right),
$$

and the transmissivity is

$$
t=\tanh (\beta J) \equiv \frac{B}{A}
$$

For Fig. 1(b) (series array) we have

$$
\begin{aligned}
e^{-\beta \mathscr{H} \mathbb{1 S}_{12}} & =\sum_{S_{3}} e^{-\beta \mathscr{H}_{123}}=\sum_{S_{3}} e^{-\beta\left[J_{1} S_{1} S_{3}+J_{2} S_{3} S_{2}\right]} \\
& =\cosh \left(\beta J_{1}\right) \cosh \left(\beta J_{2}\right)\left[1+S_{1} S_{2} \tanh \left(\beta J_{1}\right) \tanh \left(\beta J_{2}\right)\right],
\end{aligned}
$$

which exemplifies the series composition algorithm

$$
t_{s}=t_{1} t_{2} .
$$

Consider now the parallel array (Fig. 1(c)). The Boltzmann factor is given by

$$
e^{-\beta \mathscr{H}_{12}^{(p)}}=e^{\beta\left[J_{1} S_{1} S_{2}+J_{2} S_{1} S_{2}\right]}=e^{\beta J_{p} S_{1} S_{2}},
$$

where $J_{p}=J_{1}+J_{2}$. The associated transmissivity $t_{p}$ is given by

$$
t_{p}=\tanh \left(\beta J_{p}\right)=\tanh \left[\beta J_{1}+\beta J_{2}\right]=\frac{\tanh \left(\beta J_{1}\right)+\tanh \left(\beta J_{2}\right)}{1+\tanh \left(\beta J_{1}\right) \tanh \left(\beta J_{2}\right)} ;
$$

hence

$$
t_{p}=\frac{t_{1}+t_{2}}{1+t_{1} t_{2}}
$$

which is the parallel composition algorithm. Eq. (11) can be rewritten as follows:

$$
\frac{1-t_{p}}{1+t_{p}}=\frac{1-t_{1}}{1+t_{1}} \frac{1-t_{2}}{1+t_{2}} .
$$


The dual transmissivity $t^{D}[1,27]$ is

$$
t^{D} \equiv \frac{1-t}{1+t}
$$

so

$$
t_{p}^{D}=t_{1}^{D} t_{2}^{D}
$$

which presents the parallel composition algorithm in the form of the series one.

The square lattice is self-dual. Using this fact, Kramers and Wannier [28] showed that the exact critical temperature for the Ising ferromagnet is given by $t_{c}=\sqrt{2}-1$ (so, $k_{B} T_{c} / J=2.269 \ldots$ ). The graph dual of that of Fig. 1a is indicated in Fig. 2(a). Consequently, the critical point satisfies

$$
t_{c}^{D}=t_{c}
$$

which implies

$$
\frac{1-t_{c}}{1+t_{c}}=t_{c}
$$

hence $t_{c}=\sqrt{2}-1$.

We repeat the procedure for the series array (see Fig. 2(b)). We obtain

$$
t_{c}^{2}=\frac{2 t_{c}^{D}}{1+\left[t_{c}^{D}\right]^{2}}
$$

which, once again, recovers the exact result $t_{c}=\sqrt{2}-1$. If we used here the same procedure for increasingly larger clusters, we would always recover the exact answer $t_{c}=\sqrt{2}-1$. We then see that the duality is a fundamental property, and that the transmissivity variable simplifies the calculation.

In the next section, we shall follow these two different paths (single bond and series array of bonds) just illustrated to extend this simple duality argument to arbitrary values of $q$; by simple we mean that it has not been necessary to consider the entire square lattice as in the original paper by Kramers and Wannier [28], who used duality arguments to derive $t_{\mathfrak{c}}=\sqrt{2}-1$.
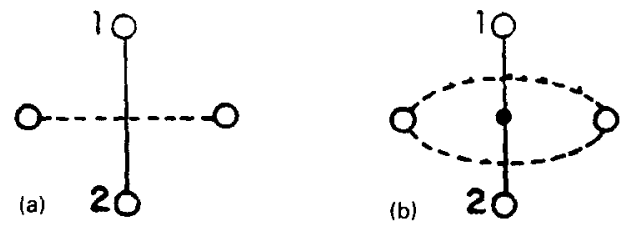

Fig. 2. Single (a) and two-series (b) bond arrays (solid lines), and their respective dual clusters (dashed lines). 


\section{General- $q$ Tsallis statistics}

We follow here the above procedure, replacing in the proper place the Boltzmann Gibbs statistics $(q=1)$ by the general $q$ result. For the Hamiltonian associated with Fig. 1(a)

$$
-\beta \mathscr{H}=K_{0}+K S_{1} S_{2}
$$

where $K \equiv B J$ and $K_{0}$ is an additive constant. Eq. (6) yields

$$
\left[1+(1-q)\left(K_{0}+K S_{1} S_{2}\right)\right]^{1 /(1-q)}=A+B S_{1} S_{2}=A\left(1+\frac{B}{A} S_{1} S_{2}\right)
$$

which yields the general transmissivity given by Eq. (7), $t \equiv B / A$, as before, where

$$
\begin{aligned}
& A \equiv \frac{1}{2}\left\{a^{+}+a^{-}\right\}, \\
& B \equiv \frac{1}{2}\left\{a^{+}-a^{-}\right\},
\end{aligned}
$$

with

$$
\begin{aligned}
& a^{+} \equiv\left[1+(1-q)\left(K_{0}+K\right)\right]^{1 /(1-q)}, \\
& a^{-} \equiv\left[1+(1-q)\left(K_{0}-K\right)\right]^{1 /(1-q)} .
\end{aligned}
$$

We recover the transmissivity $t=\tanh K$ when $q \rightarrow 1$. When the argument is negative the probability is to be taken zero [4].

We assume that Eq. (12) continues to be valid (at least as an approximation) for the generalized transmissivity $t(q)$. The self-duality of the square lattice implies $t_{c}^{D}(q)=$ $t_{c}(q)$ for the critical point, or

$$
\frac{1-t_{c}(q)}{1+t_{c}(q)}=t_{c}(q)
$$

Consequently, the critical point is $t_{c}(q)=\sqrt{2}-1$, which implies

$$
\frac{a^{+}-a^{-}}{a^{+}+a^{-}}=\sqrt{2}-1 \text {. }
$$

We present now the critical point for three cases, namely, $K_{0}=0, K_{0}=K$ and $K_{0}=-K$. For $K_{0}=0$ the critical point is given by

$$
K_{c}^{-1}=(1-q) \frac{1+(\sqrt{2}-1)^{1-q}}{1-(\sqrt{2}-1)^{1-q}}
$$

In Fig. 3 we represent $K_{c}^{-1}$ as a function of $q$.

For $K_{0}=K$ we obtain, for the critical point,

$$
K_{c}^{-1}=\frac{2(1-q)}{(\sqrt{2}+1)^{1-q}-1} .
$$




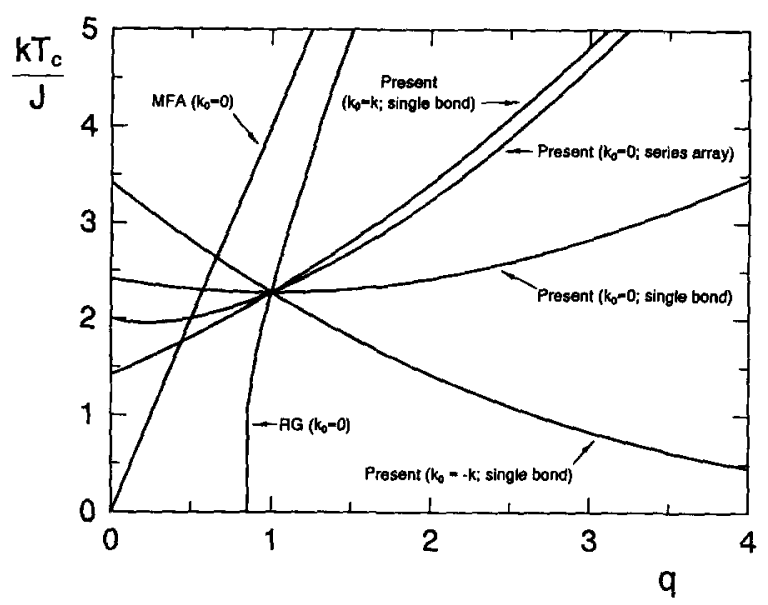

Fig. 3. Dimensionless critical temperature $k T_{c} / J$ versus $q$ for six different cases/approximations (we indicate the corresponding $q \rightarrow \infty$ asymptotic behavior): present single bond for $K_{0}=0\left(k T_{c} / J \sim q\right)$; present single bond for $K_{0}=K\left(k T_{c} / J \sim 2 q\right)$; present single bond for $K_{0}=-K$.

In Fig. 3 we plot the corresponding function $K_{c}^{-1}$ versus $q$. The third case, $K_{0}=-K$, yields, for the critical point,

$$
K_{\mathfrak{c}}^{-1}=\frac{2(1-q)}{1-(\sqrt{2}-1)^{1-q}},
$$

which is also shown in Fig. 3.

We see that all these cases reduce to the exact result for $q=1$. For $q \neq 1$ they differ among them. For the $K_{0}=0$ case, we can compare the present results with other kinds of solution such as mean field approximations (MFA) [20] and renormalization group (RG) [29].

Let us now consider the $K_{0}=0$ case associated with the series array given by Fig. 1(b). The equivalent single bond graph is obtained as follows:

$$
\begin{aligned}
{[1} & \left.+(1-q)\left(K_{0}^{s}+K_{s}\right) S_{1} S_{2}\right]^{1 /(1-q)} \\
& =\sum_{S_{3}}\left[1+(1-q) K\left(S_{1} S_{3}+S_{3} S_{2}\right)\right]^{1 /(1-q)} \\
& =\left[1+(1-q) K\left(S_{1}+S_{2}\right)\right]^{1 /(1-q)}+\left[1-(1-q) K\left(S_{1}+S_{2}\right)\right]^{1 /(1-q)} \\
& \equiv C+D S_{1} S_{2}=C\left[1+\frac{D}{C} S_{1} S_{2}\right]
\end{aligned}
$$

For $S_{1}=S_{2}=1$ we obtain

$$
[1+2(1-q) K]^{1 /(1-q)}+[1-2(1-q) K]^{1 /(1-q)}=C+D .
$$


For $S_{1}=-S_{2}=1$ we obtain

$$
2=C-D \text {. }
$$

From (30) and (31) we determine $C$ and $D$, and find

$$
t_{s}(q) \equiv \frac{D}{C}=\frac{[1+2(1-q) K]^{1 /(1-q)}+[1-2(1-q) K]^{1 /(1-q)}-2}{[1+2(1-q) K]^{1 /(1-q)}+[1-2(1-q) K]^{1 /(1-q)}+2}
$$

For the parallel array given in Fig. 1(c) we have, for the equivalent single bond cluster (by using Eq. (19) for $K_{0}=0$ ),

$$
\begin{aligned}
\left.1+(1-q) K_{p} S_{1} S_{2}\right]^{1 /(1-q)} & =\left[1+2(1-q) K S_{1} S_{2}\right]^{1 /(1-q)} \\
& =A(2 K)\left[1+t_{p}(q) S_{1} S_{2}\right],
\end{aligned}
$$

with

$$
t_{p}(q) \equiv \frac{[1+2(1-q) K]^{1 /(1-q)}-[1-2(1-q) K]^{1 /(1-q)}}{[1+2(1-q) K]^{1 /(1-q)}+[1-2(1-q) K]^{1 /(1-q)}}
$$

The duality relation we are now considering is given by

$$
t_{s}(q)=\frac{1-t_{p}(q)}{1+t_{p}(q)}
$$

which yields, for the critical point,

$$
\left[1+2(1-q) K_{c}\right]^{1 /(1-q)}-\left[1-2(1-q) K_{c}\right]^{1 /(1-q)}=2 .
$$

In Fig. 3 we present $K_{c}^{-1}$ versus $q$.

We verify in the $K_{0}=0$ case that while the $q=1$ discussion provides the exact answer independently of the size of the clusters, this is not for $q \neq 1$. Consequently, larger clusters should be investigated in order to improve the present approximations.

\section{Discussion}

Following the previous lines $[1,2]$ we generalize, within the Tsallis statistics, the transmissivity variable associated with the spin $\frac{1}{2}$ Ising ferromagnet. Then we use duality arguments in order to approach the critical temperature of this model for arbitrary $q$. Our main conclusions are:

(i) The critical temperature generically depends, as expected, on the value of the additive constant introduced in the Hamiltonian (this effect is suppressed only for $q=1$ ). This result reinforces the same conclusion obtained within renormalization group techniques [29].

(ii) The discussion of the $K_{0}=0$ case illustrates the care that must be taken when doing approximations for $q \neq 1$. For example, all the approximations presently available yield, in the $q \rightarrow \infty$ limit, $k_{B} T_{c} / J \sim b q$, but great uncertainty is observed 
concerning the prefactor $b ; b=1$ within the present single bond approximation and $b=\frac{1}{2}$ within the series array approximation, to be compared with $b=4$ for the mean field approximation [20] and $b=5.5$ for a renormalization group approximation [29]. The sensitivity of the results on the size of the clusters involved in the approximation is reminiscent of the situation observed in quantum systems [30].

The present results could be useful in the discussion of magnetism in fractal structures where standard (extensive) thermodynamics are expected to fail. Further studies (like the present approach but using larger clusters, Monte Carlo techniques, and others) are welcome in order to better understand the influence of $q$ on critical points.

\section{Acknowledgements}

This work was partially supported by grants from $\mathrm{CNP}_{\mathbf{q}}$.

\section{References}

[1] C. Tsallis and S.V.F. Levy, Phys. Rev. Lett. 47 (1981) 950.

[2] J.M. Maillard, F.Y. Wu and Chin-Kun Hu, J. Phys. A 25 (1992) 2521; C. Tsallis and A.C.N. de Magalhães, Phys. Rep. 268 (1996) 305.

[3] J.W. Essam and C. Tsallis, J. Phys. A 19 (1986) 411; A.C.N. de Magalhães and J.W. Essam, J. Phys. A 19 (1986) 1655; A.C.N. de Magalhães and J.W. Essam, J. Phys. A 21 (1988) 473.

[4] C. Tsallis, J. Stat. Phys. 52 (1988) 479; E.M.F. Curado and C. Tsallis, J. Phys. A 24 (1991) L69; Errata: J. Phys. A 24 (1991) 3187; E.M.F. Curado and C. Tsallis, J. Phys. A 25 (1992) 1019; C. Tsallis, Phys. Lett. A 206 (1995) 389.

[5] P.A. Alemany and D.H. Zanette, Phys. Rev. E 49 (1994) R956; D.H. Zanette and P.A. Alemany, Phys. Rev. Lett. 75 (1995) 366; C. Tsallis, S.V.F. Levy, A.M.C. de Souza and R. Maynard, Phys. Rev. Lett. 75 (1995) 3589; see also Eq. (5) of O.V. Bychuk and B. O'Shaughnessy, Phys. Rev. Lett. 74 (1995) 1795.

[6] A.R. Plastino and A. Plastino, Physica A 222 (1995) 347; C. Tsallis and D.J. Bukman, Phys. Rev. E 54 (1996) R2197; see also H. Spohn, J. Phys. I France 3 (1993) 69.

[7] A.R. Plastino and A. Plastino, Phys. Lett. A 174 (1993) 384; J.J. Aly, in: Proc. Meeting on N-Body Problems and Gravitational Dynamics, eds. F. Combes and E. Athanassoula (Aussois, France, 21-25 March 1993), (Publications de l'Observatoire de Paris, Paris, 1993) p. 19; A.R. Plastino and A. Plastino, Phys. Lett. A 193 (1994) 251.

[8] B.M. Boghosian, Phys. Rev. E 53 (1996) 4754.

[9] P. Jund, S.G. Kim and C. Tsallis, Phys. Rev. B 52 (1995) 50.

[10] L.S. Lucena, L.R. da Silva and C. Tsallis, Phys. Rev. E 51 (1995) 6247.

[11] C. Tsallis, F.C. Sá Barreto and E.D. Loh, Phys. Rev. E 52 (1995) 1447.

[12] T.J.P. Penna, Phys. Rev. E 51 (1995) R1; D.A. Stariolo and C. Tsallis, Ann, Rev. Comp. Phys., Vol. II, eds. D. Stauffer (World Scientific, Singapore, 1995) p. 343; T.J.P. Penna, Comput. in Phys. 9 (1995) 341; K.C. Mundim and C. Tsallis, Int. J Quantum Chem. 56 (1996) 373; J. Schulte, Phys. Rev. E 53 (1996) 1348

[13] A.M. Mariz, Phys. Lett. A 165 (1992) 409.

[14] J.D. Ramshaw, Phys. Lett. A 175 (1993) 169.

[15] J.D. Ramshaw, Phys. Lett. A 175 (1993) 171.

[16] A.R. Plastino and A. Plastino, Phys. Lett. A 177 (1993) 177.

[17] A.R. Plastino and A. Plastino, Physica A 202 (1994) 438. 
[18] A. Plastino and C. Tsallis, J. Phys. A 26 (1993) L893.

[19] D.A. Stariolo, Phys. Lett. A 185 (1994) 262.

[20] E.F. Sarmento, Physica A 218 (1995) 482.

[21] A. Chame and E.V.L. de Mello, J. Phys. A 27 (1994) 3663; M.O. Caceres, Physica A 218 (1995) 471.

[22] C. Tsallis, in: New Trends in Magnetism, Magnetic Materials and Their Applications, eds. J.L. Morán López and J.M. Sánchez (Plenum Press, New York, 1994) p. 451; see also C. Tsallis, Chaos, Solitons and Fractals 6 (1995) 539.

[23] C. Tsallis, Phys. Lett. A 195 (1994) 329.

[24] E.P. da Silva, C. Tsallis and E.M.F. Curado, Physica A 199 (1993) 137; Erratum: Physica A 203 (1994) 160.

[25] R.F.S. Andrade, Physica A 175 (1991) 185.

[26] R.F.S. Andrade, Physica A 203 (1994).

[27] A.C.N. de Magalhães, J.W. Essam and F.Y. Wu, J. Phys. A 23 (1990) 2651.

[28] H.A. Kramers and G.H. Wannier, Phys. Rev. 60 (1941) 252.

[29] S.A. Cannas and C. Tsallis, Z. Phys. B 100 (1996) 623.

[30] A.O. Caride, C. Tsallis and S.F. Zanette, Phys. Rev. Lett. 51 (1983) 145; Phys. Rev. Lett. 51 (1983) 616; A.M. Mariz, C. Tsallis and A.O. Caride, J. Phys. C 18 (1985) 4189. 